Revue d'histoire de l'Amérique française

BRS REVUE D.HISTOIRE DE L'AMÉRIQUE FRANÇAISE

\title{
Les problèmes historiques de la langue franco-canadienne
}

\section{Gaston Dulong}

Volume 10, numéro 1, juin 1956

URI : https://id.erudit.org/iderudit/301743ar

DOI : https://doi.org/10.7202/301743ar

Aller au sommaire du numéro

Éditeur(s)

Institut d'histoire de l'Amérique française

ISSN

0035-2357 (imprimé)

1492-1383 (numérique)

Découvrir la revue

Citer cet article

Dulong, G. (1956). Les problèmes historiques de la langue franco-canadienne. Revue d'histoire de l'Amérique française, 10(1), 69-76.

https://doi.org/10.7202/301743ar d'utilisation que vous pouvez consulter en ligne.

https://apropos.erudit.org/fr/usagers/politique-dutilisation/ 


\section{LES PROBLĖMES HISTORIQUES DE LA LANGUE FRANCO-CANADIENNE *}

Toute langue pose des problèmes historiques avec lesquels linguistes, philologues et dialectologues sont aux prises et auxquels ils essaient d'apporter des réponses précises. La langue franco-canadienne ne fait pas exception à la règle générale. Elle a aussi ses problèmes historiques.

Notre intention, dans cette brève causerie, n'est pas de faire le relevé exhaustif de ces problèmes mais d'en soulever quelquesuns pour ensuite essayer de montrer les conséquences pratiques de ces mêmes problèmes pour le dialectologue ou pour l'historien de la langue franco-canadienne.

Le premier problème important est certes celui du peuplement. D'où venaient les colons français qui s'établirent en Nouvelle-France aux $17^{\mathrm{e}}$ et $18^{\mathrm{e}}$ siècles ? Des généalogistes consciencieux se sont attaqués à ce problème (citons en passant Lortie, Massicotte et le Père Godbout). Leurs dépouillements révèlent que certaines anciennes provinces françaises comme la Normandie, l'Île-de-France, le Poitou et les provinces environnantes ont fourni une part importante du contingent de colons qui se sont fixés sur les rives du Saint-Laurent. Il est sûr, d'autre part, que les colons venus s'établir ici au $17^{\circ}$ siècle ont joué dans la formation de la langue franco-canadienne un rôle plus important que ceux du siècle suivant.

Le même problème se pose pour le peuplement de l'Acadie. Je connais très mal les travaux des généalogistes sur le peuplement de l'Acadie. Cependant une chose semble de plus en plus assurée: aujourd'hui, l'étude de la langue acadienne indique, d'une façon très nette, que les ancêtres des Acadiens venaient surtout de l'Ouest de la France: Poitou, Aunis et Saintonge.

* Travail présenté à la Réunion générale de l'Institut d'histoire de l'Amérique française, le 14 avril 1956. 
Le corollaire obligé de l'origine des colons venus s'établir ici est leur répartition en Nouvelle-France. Par exemple, les habitants des alentours de Québec furent-ils plus normands que ceux de la région de Montréal ? Viennent-ils davantage de l'Ouest de la France, c'est-à-dire du Poitou, de l'Aunis et de la Saintonge ? A une époque beaucoup plus proche de nous, le fait que le $L a c$ Saint-Jean ait été colonisé surtout par des gens venant du comté de Charlevoix, que la côte nord de la Gaspésie l'ait été surtout par des gens de Montmagny, de Lislet et de Kamouraska, que les Cantons de l'Est (ou pour employer un néologisme toponymique, l'Estrie) l'aient été par des gens venant de la Beauce, de Lotbinière, de Nicolet, de Yamaska ... etc., voilà autant de problèmes historiques dont doit tenir compte l'historien de la langue francocanadienne.

La conquête de 1760 suivie de la cession du Canada à l'Angleterre en 1763 est un autre événement historique dont les conséquences pour notre langue se font encore sentir. Les contacts avec la France cessent à peu près complètement; l'immigration de langue française est tarie; nos villes sont occupées par l'armée anglaise; l'administration est anglaise; le droit criminel et le droit commercial sont anglais; le commerce passe entre les mains de gens de langue anglaise, des immigrants de langue anglaise s'installent à demeure au milieu de nous.

$\mathrm{Au} 19^{\mathrm{e}}$ siècle s'amorce lentement une révolution industrielle qui aujourd'hui n'est pas encore arrivée à son terme. Nos gens doivent, pour se procurer leur pain quotidien, travailler de plus en plus nombreux pour des étrangers, sous des contremaîtres de langue anglaise. Les métiers traditionnels tombent peu à peu en désuétude; l'agriculture se mécanise; l'électrification et la radio, aujourd'hui la télévision, atteignent les campagnes les plus reculées. Et chaque innovation apporte son contingent de mots nouveaux, la plupart du temps étrangers, anglais ou américains.

Un autre facteur ne doit pas être oublié pour expliquer l'invasion des mots étrangers dans la langue franco-canadienne: c'est l'aspect vagabond de la vie d'une partie de la population. Pendant tout le $19^{\circ}$ siècle beaucoup de Canadiens français sont devenus forestiers et voyageurs à la solde des compagnies de 
fourrures ou des compagnies forestières. De plus, à partir de 1850, un nombre toujours croissant des nôtres allèrent s'installer aux États-Unis: quelques-uns revinrent dans le Québec avec un petit pécule; la plupart s'installèrent à demeure outre-frontières conservant des relations familiales avec ceux qui étaient restés ici.

Voilà autant de problèmes historiques ou de facteurs qui ont contribué à l'élaboration et à la transformation de la langue franco-canadienne, telle que nous pouvons la recueillir aujourd'hui sur les lèvres d'un homme du peuple. Et qu'on n'aille pas croire qu'il existe de ces régions privilégiées qui n'auraient pas subi les répercussions des facteurs que je viens d'énumérer. Il se produit dans les langues ce phénomène bien connu de l'osmose qui apparaît partout et dont seule l'intensité peut varier d'une région à une autre.

Les conséquences pratiques pour l'histoire de la langue franco-canadienne des problèmes que nous venons de signaler sont extrêmement importantes. La première de ces conséquences, c'est la langue que parlaient les colons à leur arrivée au Canada. Rivard dans Étude sur les parlers de France au Canada a très bien répondu à cette question. Les colons parlaient sans aucun doute le patois de leur province d'origine, tout en ayant une certaine connaissance de la langue commune, le français. Les archives conservent sans doute plusieurs preuves de cela. En voici deux dont la première seule était connue de Rivard. Dans un procès entre deux habitants de la seigneurie de Lauzon, et dont le procès-verbal se trouve dans les archives de la Prévôté de Québec pour les années 1666 à 1669, un témoin rapporte une conversation entre le demandeur et le défendeur; à un certain moment, le récit est interrompu: le témoin n'a pu comprendre le reste du discours, car le défendeur «parlait dans son patois ». Autre preuve que m'a signalé mon ami Marcel Trudel et concernant l'Acadie cette fois. En 1718, Le Conseil de la Marine fait passer en Acadie un prêtre de Bayonne pour desservir les Basques qui ne comprenaient pas le français (Le Conseil à SaintOdile, 4 mai 1718: RAC, 1899, supplément: 515). A la fin du régime français, le Père Potier, missionnaire jésuite, a écrit un manuscrit renfermant les Façons de parler proverbiales, tri- 
viales, figurées ... des Canadiens $d u 16^{e}$ siècle. Or ce manuscrit, publié dans le BPFC en 1905-1906 renferme beaucoup de mots patois encore en usage ici. Voici quelques exemples :

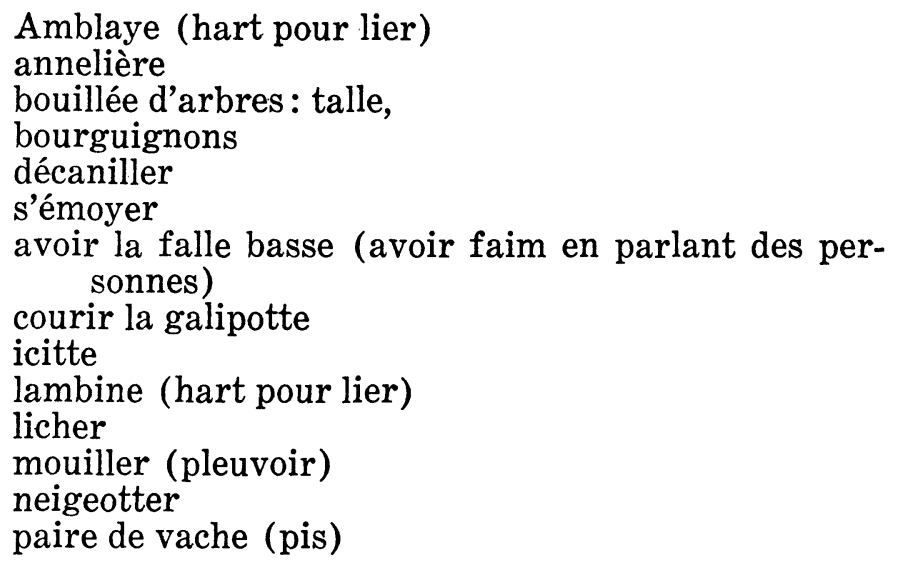

La liste de plus de 1,000 mots ou expressions de Potier renferme aussi des mots patois qui semblent avoir disparu depuis, dont en tout cas, on n'a pas encore retracé la survivance. C'est le cas de

breuilles (entrailles)

ébreuiller (étriper, vider de ses entrailles)

Si l'on n'était pas convaincu que les colons venus ici parlaient sans doute le patois, rappelons les conclusions de l'enquête par correspondance de l'abbé Grégoire, menée à travers toute la France en 1790 et 1791, pour se renseigner sur l'état des patois. Cette enquête, dont le rapport fut présenté au Comité de l'instruction publique en 1794, révèle l'existence de trente patois rappelant les anciennes provinces de France. «On peut assurer, continue le rapport, sans exagération, qu'au moins six millions de Français, surtout dans les campagnes, ignorent la langue nationale; qu'un nombre égal est à peu près incapable de soutenir une conversation en français; qu'en dernier résultat, le nombre de ceux qui la parlent purement n'excède pas 3 millions, et probablement le nombre de ceux qui l'écrivent correctement est encore moindre. »

Cela, 40 ans après la fin du régime français au Canada. 
Pour nous convaincre davantage du fait que le patois fut parlé ici, rappelons aussi, qu'on a même recueilli au moins une chanson en patois jersais au Canada. Cette chanson fut recueillie en 1866, dans le comté de Bonaventure, et publiée la même année avec une traduction française, dans Le Foyer canadien, tome IV, pages 396-398.

D'ailleurs, le Glossaire du Parler français au Canada publié en 1930 par la Société du Parler français, à la suite d'une enquête par correspondance qui a duré environ 15 ans (de 1902 à 1917), renferme plusieurs milliers de mots patois. Oh, je sais bien que plusieurs croient plus honorable de répéter que nos ancêtres parlaient la langue de Racine et de Bossuet. Il est plus facile de parler de pauvreté de vocabulaire, de déformations phonétiques là ou le chercheur consciencieux retrouve des éléments patois ou de langue populaire importés de France par les colons venus s'établir ici.

J'ouvre ici une parenthèse pour dire que notre langue ne se compose pas exclusivement de ces éléments patois ou populaires mais comporte en même temps une part respectable de la langue française commune, de la langue de culture. C'est que, dès leur arrivée ici, les immigrants patoisants ont dû apprendre très tôt au moins les rudiments du français. Imaginez, en effet, un patoisant poitevin ayant à s'entretenir avec un patoisant normand ou picard. Si chacun des interlocuteurs parle son patois, il leur sera impossible de converser. D'un commun accord, ils essaieront de se faire comprendre en se servant des rudiments de français qu'ils possèdent déjà. Tout, par ailleurs, milite en faveur de la langue commune: le français est la langue de l'administration, de l'armée, de l'église ... Les patoisants garderont de leur patois importé des intonations, des tournures syntaxiques, des façons de prononcer, surtout des mots, ceux de tous les jours, mots humbles servant à désigner leurs outils, leurs façons de travail, ce qui a trait à leurs bêtes. Et, semble-t-il, lorsque dans une région donnée, l'élément poitevin, par exemple, aura joué dès le début un rôle prédominant, le mot poitevin éliminera les autres mots patois. On ne peut expliquer autrement la localisation géographique des mots. 


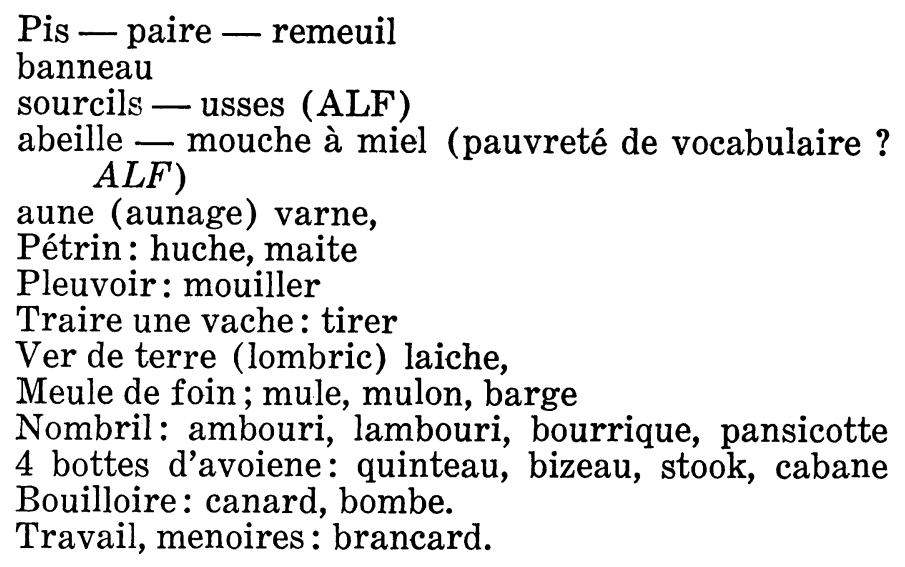

Comment expliquer l'emploi de tel mot dans une région et de tel autre dans une autre ? Sinon que ce mot, dès le début, a éliminé les autres mots patois, parce que sans doute employé par un plus grand nombre de sujets parlants.

L'histoire de la langue franco-canadienne doit se faire en partant de l'observation directe de cette langue telle qu'elle est parlée aujourd'hui, c'est-à-dire par l'enquête sur le terrain. C'est là un point sur lequel je n'insisterai pas devant vous.

La langue franco-canadienne, nous la trouvons aussi dans les textes anciens, manuscrits ou imprimés: c'est pourquoi, l'historien de la langue franco-canadienne doit avoir accès aux sources au lieu de se contenter de la solution facile de répéter ce que d'autres ont dit avant lui. Le dépouillement des sources en vue de connaître notre langue est extrêmement fructueux et éclaire singulièrement l'histoire de nos mots, aide beaucoup à composer ce que j'appelle l'état civil de chaque mot. Quelques exemples pour illustrer.

Poudrerie: canadianisme, Begon, Potier 1743, R.J. 1709 par le père Silvy,

Dictionnaire françois-onontagué, édité par Shea à N.-Y. en 1859 d'après un mss de la fin du $17^{\mathrm{e}}$ siècle

Sagard, dans son Histoire du Canada (1636) et dans son Dictionnaire de la langue huronne, ignore poudrerie et poudrer et emploie la périphrase « La neige voltige en poussière » 
Cageux (radeau) Canadianisme ?

Journal du Chevalier La Pause: 1759

Potier: 1743

Ordonnance de Hocquart: 1731

Lettre de Vaudreuil et Bégon au Conseil de Marine: 1716

Comptes du Séminaire de Québec en 1693

(Chose et mot connus depuis longtemps;

Mitaines que quelques-uns croient faire venir de l'anglais

Courant dans des textes du Régime français (actes de donation)

Carriole (avec sens canadien)

Description très détaillée dans Franquet,

apparaît aussi avant, v: Correspondance de Madame Bégon

Tuque employé sous le régime français.

Chanson imprimée de 1758

Grands services des dépouillements pour les mots empruntés aux langues indiennes. Certain nombre sont passés au français commun. On peut les faire remonter bien antérieurement à ce qu'indiquent les dictionnaires étymologiques du français.

Sagamité (début $19^{\mathrm{e}}$ s., Chateaubriand)

Or apparaît déjà dans Sagard: Dictionnaire de la langue huronne, 1632, Histoire du Canada (1636).

Carcajou (Dictionnaire étymologique de Von Wartburg (1751); Dauzat, Supplément (1710).

Or déjà dans La Hontan: 1705

Nicolas Denys : 1672

Même chose pour Manitou, Pirogue et plusieurs autres.

Une connaissance plus précise de l'emploi de beaucoup de mots indiens dans les textes anciens aurait sans doute rendu nos épureurs de langage un peu moins sévères. Par exemple ils n'auraient pas vu d'anglicisme dans l'emploi du mot tobagane ou dans le mot mahogany (acajou) mots d'ailleurs acceptés par l'américain et l'anglo-canadien.

Ce recours aux textes aide à éclairer l'histoire de notre langue, même pour ce qui a trait aux anglicismes. Par exemple a-t-on remarqué que l'emploi pourtant du mot français mille 
(mesure de longueur) a été répandu sous le régime anglais, ce mot n'apparaissant jamais, semble-t-il, dans les textes du régime français ?

Comment s'est faite la lutte entre piastre et dollar? Comment se fait-il que ce nouveau venu, dollar, a réussi à éliminer son concurrent dans le langage officiel ? Vers quelle date?

A quelle époque précise drave et draver, draveur, ont-ils fait leur apparition?

D'un autre côté souvent nous avons dû donner à des mots déjà français une acception qu'ils n'avaient pas dans le français commun: c'est le cas de canton (mot lancé semble-t-il par GérinLajoie) et qui a fini par éliminer township. C'est aussi le cas du mot comté qui n'a en français commun qu'un sens historique.

Vous avez là une partie du travail considérable du dialectologue qui s'intéresse à la langue franco-canadienne. Nous avons parlé de la naissance des mots dans des temps parfois anciens, mais tous les jours nous assistons à la naissance de nouveaux mots qu'il faut consigner pour la postérité, mots tels que motel, centre d'achat, auto-banque...

Il faut aussi surveiller la disparition des mots qui meurent, car souvent ils continuent de vivre dans des expressions stéréotypées, la plupart du temps figurées. Les ptits chars sont déjà disparus à Québec depuis la fin de la guerre et sont à la veille de disparaître dans la Métropole. La chose disparaissant, le mot disparaîtra aussi. Mais peut-être continueront-ils de vivre dans un emploi figuré comme milieu-de-canot appliqué à un paresseux ou une Croix de saint-Louis employé dans l'expression, ce n'est pas une croix de saint-Louis, en parlant de quelqu'un qui n'est pas très brillant ou qui est d'une moralité peu reluisante.

Pour mener à bien ce travail sur la langue franco-canadienne, il faudra encore beaucoup de travail, beaucoup d'enquêtes, de dépouillements de textes. Et je pense ici à la collaboration précieuse que pourraient et que voudront sans doute apporter les historiens, à l'édification d'un dictionnaire de notre langue, dictionnaire qui ne pourra être qu'une œuvre de collaboration.

Gaston DULONG, Professeur à l'Université Laval, (Québec). 